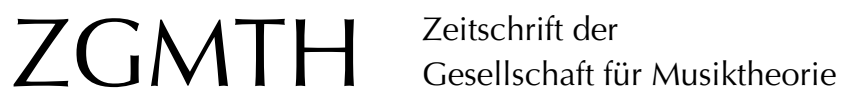

Holtmeier, Ludwig (2003/05): Zum Dresdner Promotionsrecht. ZGMTH 1-2/1/1, 137146. https://doi.org/10.31751/487

(C) 2003/05 Ludwig Holtmeier

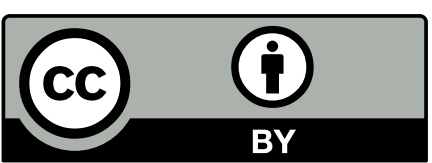

Dieser Text erscheint im Open Access und ist lizenziert unter einer Creative Commons Namensnennung 4.0 International Lizenz.

This is an open access article licensed under a

Creative Commons Attribution 4.0 International License.

veröffentlicht / first published: 01/01/2003

zuletzt geändert / last updated: 15/09/2009 


\section{Zum Dresdner Promotionsrecht}

Nur einige Tage nach dem großen Erfolg des 1. Kongresses der Deutschen Gesellschaft für Musiktheorie wurde in Dresden (2001) beschlossen, das Promotionsrecht im Fach Musiktheorie einzuführen. Folgender Aufruf wurde nach dem ebenso enthusiastisch aufgenommenen 2. Kongreß in München (2002) an viele Kolleginnen und Kollegen verschickt. Die folgende Liste von Meinungen sei als offene verstanden, die der Fortsetzung harrt.

Liebe Kollegen, Mitglieder und Freunde der GMTH, nach dem großen Erfolg unseres diesjährigen Münchner Kongresses möchte ich Sie zur Beteiligung an einem kleinen Projekt aufrufen. Wie Sie wissen, ist unmittelbar nach dem letztjährigen Dresdner Kongreß das erste gänzlich eigenständige Promotionsrecht im Fach Musiktheorie in Dresden eingeführt worden. Was bei den einen große Begeisterung hervorruft, wird von anderen kritisch betrachtet, von einigen sogar unumwunden abgelehnt. Wie immer man zu dieser Entwicklung auch stehen mag: Sie stellt einen Meilenstein in der Institutionengeschichte unseres Faches dar. Es war ein shistorischer Moments, ein big point in unserer kleinen, unbedeutenden Fachgeschichte. Ob diese Entwicklung dem Fach zum Guten oder zum Schlechten gereichen wird, welche Chancen und welche Gefahren dadurch eröffnet werden, dazu möchten wir Sie befragen. Weder dem Umfang noch der Form ihres Kommentars sind dabei Grenzen gesetzt. In der Hoffnung, von Ihnen zu hören, verbleibe ich mit freundlichen Grüßen

Ludwig Holtmeier

\section{Allen Forte, Battell Professor of Theory of Music, Yale University Fellow, American Academy of Arts and Sciences}

(Allen Fortes Kommentar bezieht sich auf die Gründung der GMTH)

This is in response to your email letter of October 16 in which you invite opinions of the plan to form an official German society devoted to music theory. I am happy to respond and to offer my view from my background as first President of the Society for Music Theory. While I deplore the negative reaction you have received to this project from a segment of the German scholarly community, I am not really surprised. Indeed, it seems all too familiar, since the Society for Music Theory elicited a similar response at the time it was formed in 1977. At the banquet in celebration of the twenty-fifth anniversary of the founding of the society, held in Columbus, Ohio on November 2, I spoke of the events attending that momentous event, including the opposition the founders encountered from certain members of the American Musicological Society. Nevertheless, we moved forward, established a journal (Music Theory Spectrum), and initiated annual meetings, 
among other professional activities. Almost from the very beginning we had a thriving, enthusiastic membership, and we enjoy a wonderful collegiality and a very fine relation with our sister society, the American Musicological Society, whose membership intersects with ours in a number of instances (mine, for example). With this background in mind, I can only encourage you to move ahead in the formation of the new German society. I join Professor Thomas Christensen in his support of this initiative and in his general and admirable interest in forging international connections among those of us deeply involved in music-theoretic research and related projects. In offering my support for this endeavor I cannot refrain from stating the obvious, namely, that a German society dedicated to music theory will be a noble successor to the ancient tradition of major writings by authors whose roots are embedded in the culture of Germany. Names such as those of Heinichen and Kirnberger spring to mind. And there are many more, as you well know.

\section{Jürg Stenzl, Salzburg}

Viel brauche ich dazu nicht zu sagen: Da in den USA »Music Theory« und »Music Analysis« längst Promotionsstatus - gerade auch für angehende Komponisten - haben, war es an der Zeit. Nicht, weil die Amis alles besser machen, sondern weil es auch bei uns Arbeiten, darunter ein paar hervorragende Arbeiten, gibt, die nicht in einem - wie auch immer gearteten - Sinne primär shistorisch، ausgerichtet sind. Das gilt ganz besonders fürs 20. Jahrhundert, und hier mehr noch für dessen zweite Hälfte. Gerade weil ich Historiker bin, mich auch lebenden Komponisten gegenüber als solcher verstanden habe und verstehe, sehe ich da kein Problem, unter der Voraussetzung allerdings, daß es entsprechende methodologische Reflexionen gibt. (Aber das ist ja auch eine der schwachen Seiten der Musikhistoriker deutscher Sprache.) Um es grob, geradezu professoral zu sagen: Die Frage ist nicht, ob Historie oder Theorie, die Frage ist die Qualität. Und die - mit Verlaub - Kreativität der Arbeit und Lehre. Hier darf es allerdings keine Kompromisse geben. Die Qualität ist nicht abhängig von Universität oder Musikhochschule. (Daß - beispielsweise - die Musikhochschulen in Österreich jetzt auch Universitäten heißen, daß sich auch Instrumentalisten zuhauf habilitieren wollen - und können, ist noch kein Beweis für Offenheit und Niveau. Die guten Leute in den Musikhochschulen/ Universitäten wissen das.) Ich wünsche Ihnen und der GMTH weiterhin viel Erfolg. Es wäre schön, wenn Sie das teilweise verrottete Fach Musikwissenschaft einer partiellen Frischzellenkur unterziehen könnten.

\section{Markus Janz, Basel}

Daß der Beifall zur akademischen Würde und Er-Mächtigung auch von der falschen Seite kommen kann, ist eins (die Argumente kennen wir: mehr ist besser/jetzt sind auch wir endlich wer), das andere sind die - natürlich ebenso falschen - gegnerischen Verlautbarungen (auch die sind sattsam bekannt: Die können das doch gar nicht/das ist der Niedergang der akademischen Qualitätsstandards/da kann ja jeder kommen/anything 
goes). Ich glaube, daß für uns alle diese Ebene der Argumentation tatsächlich kein Thema sein sollte. Die Promotionsermächtigung ist eine großartige Herausforderung, ihr sollte die Sorge gelten. Am letzten Kongreß in Dresden und am eben zu Ende gegangenen Münchner Kongreß ließen sich jüngere Dozenten vernehmen, die menschlich, wissenschaftlich und künstlerisch höchst Bemerkenswertes aufzuweisen haben. Diese neue Bewegung bezieht ihre Energie natürlich aus den Personen, aber auch aus den neuen Vorgaben bezüglich der Inhalte und ihrer Gewichtung ebenso wie der Vorgehensweisen und der Kritik an diesen. Da, glaube ich, ist anzusetzen bzw. weiterzufahren. Daraus lassen sich Curricula entwickeln, davon lassen sich Zielqualitäten ableiten. Eine Aufgabe, der sich zu stellen die reine Freude sein kann, sofern man sich des eigenen Übermuts erwehren und gleichzeitig den altbacken-universitären Unmut ignorieren kann. Ob das Fach davon groß profitiert, ist für mich überflüssiges Bangen, ich finde das Hoffen und Wünschen der Menschen, der Forschenden und Lehrenden, viel interessanter. Die Bewegung wird das Fach verändern. Das wird ihm gut tun. Die höhere Anerkennung wird ihm nicht schaden. Voilà tout!

\section{Hartmut Fladt, Berlin}

Also ganz generell: wunderbar, auch nach den Erfahrungen an der Musikfakultät der UDK Berlin, wo wir de facto seit zwei Jahren die Möglichkeit haben, Promotionsverfahren im Fach »Musiktheorie« zu betreuen, wo allerdings Skeptiker aus den Bereichen Musikwissenschaft und Musikpädagogik/Didaktik, die bis dahin allein den Promotionsstudiengang betreuten, noch einmal sich um juristische Absicherung bemühten - was mir (uns) sehr recht war, denn jetzt ist seit einem Jahr das gesamte Konstrukt ıwasserdicht und von ıganz oben` abgesegnet. Allerdings geht es bei uns nicht um ein e i g e n s tä n diges Promotionsrecht, sondern: Die wissenschaftliche Musiktheorie wird als originärer Teil der Musikwissenschaft gesehen (kann in Ausnahmefällen sich sogar auch in Richtung Pädagogik/Didaktik orientieren). Das scheint mir persönlich für die Außenwirkung von musiktheoretischen Promotionen nicht nur akzeptabel zu sein, sondern verhindert die abwertende Klassifikation als Promotion zweiter Klasse - vergleichbar dem Kompositions-Doktortitel in den USA etc. Insgesamt aber ist die Chance für künstlerisch-wissenschaftliche Hochschulen/Universitäten, eine kunstnährere Wissenschaftlichkeit zu etablieren, als es den Unis jemals möglich wäre, eminent groß; daß an der UDK auch ehemalige Kompositionsstudierende (mit entsprechender musiktheoretischer Zusatzqualifikation) über kompositionsnahe Themen promovieren, finde ich natürlich wunderbar. Also: Glückwunsch - aber bitte (auch bei der Zulassung von Promotionsvorhaben) strikt auf höchste Qualität achten, so daß - gerade in der Anfangsphase - auf keinen Fall der Ruch von Zweitrangigkeit entstehen kann. Wenn ich das Niveau der Kongresse der GMTH betrachte, bin ich da allerdings zuversichtlich. 


\section{Wilfried Gruhn, Freiburg}

Gratulation zu der neu und erstmals eröffneten Möglichkeit, in Dresden im Fach Musiktheorie zu promovieren. Von der Sache her gab es ja schon immer Promotionen mit Themen aus der Musiktheorie (man denke nur an die vielen Analyse-Arbeiten zur Wiener Schule etc.). Nun soll das Fach »Musiktheorie« eigenständig neben die Musikwissenschaft und die Musikpädagogik treten. Das ist zunächst einmal für das Fach ein enormer Impuls und schließt ein, daß ıman sich stärker als theoretisch reflektierende, wissenschaftliche Disziplin verstehen möchte. In diesem Sinne versteht sich ja die Music Theory in den USA. Dagegen ist nichts einzuwenden. Aber kann das Fach das leisten, wenn nun auch andere Musikhochschulen kommen und das Promotionsrecht für Musiktheorie haben wollen? Gibt es ein personelles Reservoir an wissenschaftlichen, promovierten Musiktheoretikern? Die andere Frage ist, ob sich der wissenschaftliche Anteil der Musiktheorie, der ja schon im Namen zum Ausdruck kommt, wirklich von der Musikwissenschaft abkoppeln und eigenständig werden soll. Das muß im Fach selber diskutiert werden und wird es ja wohl auch. Ich hätte gegen eine sich wissenschaftlich verstehende autonome Disziplin »Musiktheorie« mit Promotionsrecht nichts einzuwenden.

\section{Erwin Koch-Raphael, Bremen}

Selbstverständlich stellt die Einführung des eigenständigen Promotionsrechts im Fach Musiktheorie eine als ganz und gar positiv zu bewertende Entscheidung dar. Die rasante Entwicklung im Fach Musiktheorie in den letzten Jahrzehnten, die wesentlich im Feld der historischen Musikwissenschaft liegt, läßt es nicht mehr zu, Musiktheorie zu lehren, wie es früher, als »Handwerkslehre« oder »Harmonielehre« schamhaft benannt und so verkürzt, an deutschen Musikhochschulen praktiziert wurde. Überwunden ist mit dieser Entwicklung die ohnehin längst überholte, aber noch nicht gänzlich aus der Welt genommene Kategorisierung der Musiktheorie als »Musikpraxis«, wie sie ja zum Teil heute noch - peinlicherweise - an vielen Musikhochschulen üblich ist. Was liegt also dann näher, als auch das Promotionsrecht für Musiktheorie einzuführen, will man doch die Forschung auf diesem Gebiet nicht gleich wieder zur Ruhe kommen lassen, sondern weiterführen und ermuntern, forschend dem Fach noch mehr und mehr spannende Aspekte und Einsichten in Zukunft hinzuzufügen. Ganz zu schweigen von der Aufwertung, die das Fach in der deutschen Hochschullandschaft längst verdient hat und die außerhalb Deutschlands in der EU längst weitgehend erfolgt ist. Kurzum: Gratulation für diese kluge Entscheidung, die in Dresden gefallen ist. Ich hoffe auf baldige ^Nachahmungstäter`.

\section{Dieter Mack, Lübeck}

Ich begrüße das Promotionsrecht uneingeschränkt, weil es zunächst nichts anderes bewirkt, als diesem Bereich im internationalen Vergleich ein adäquateres Daseinsrecht zuzumessen. Mit solch einer Berechtigung ist zunächst in keiner Weise eine inhaltliche Präjudizierung verbunden. Und dort scheint mir der Knackpunkt zu liegen. Die Dis- 
kussion über die inhaltlichen Bedingungen müßte noch geführt werden. Falls es zu einer Methodendiskussion à la Amerika kommen würde, hätte man meines Erachtens die Zeichen der Zeit falsch verstanden. Wenn man den Sachverhalt zum Anlaß nimmt, die bereichernde Dualität skreativ-künstlerischer Ansatz kontra Wissenschaftlichkeitı nicht 'kontraı, sondern in Kombination zu sehen, dann hätte das Fach Wesentliches beizutragen, von interdisziplinären und ethnomusikologischen Ansätzen einmal ganz abgesehen. Wenn man hingegen Musiktheorie nur als Überbegriff für einen erstarrten Fächerkanon an einer Musikhochschule begreift, dann sollte man es lassen. Aber vielleicht ist dies die Chance, diese Verkrustungen aufzuweichen.

\section{Hubert Moßburger, Bremen}

Die Frage nach der Promotion in Musiktheorie läßt sich auf die - meiner Meinung nach - rhetorische Frage reduzieren, ob der Musiktheoretiker Forschung betreiben soll oder nicht. Oder noch pointierter: Soll der in dem merkwürdig unbestimmt bleibenden Spannungsfeld zwischen Kunst und Wissenschaft befindliche, in der alltäglichen Praxis jedoch hauptsächlich lehrende Musiktheoretiker weiterhin überwiegend seine Kenntnisse aus der shöheren` Musikwissenschaft empfangen und damit lediglich in pädagogischvermittelnder Funktion auftreten? Wäre es nicht an der Zeit, das Heft selbst in die Hand zu nehmen und eigenständige musiktheoretische Forschung zu betreiben, die dann aus serster Hand vermittelt würde? Die Aktivierung solcher fachspezifischen Forschung in seigenen Angelegenheiten durch Etablierung des Pomotionsrechts würde dem Musiktheoretiker nicht nur neues Selbstvertrauen geben, sie würde gleichzeitig auch eine institutionelle Verankerung bewirken, in der Musiktheorie nicht zwischen den Fronten «Kunst und Wissenschaftı zerrieben und zum Dienstleistungsfach degradiert werden würde. Speziell musiktheoretische Forschungsaufgaben gäbe es genügend:

- Aufarbeitung historischer Theorien (über Analyse, Kompositionslehre usw.)

- Aufstellung neuer Theorien zur historischen, aber auch zur Musik des 20. und 21. Jahrhunderts

- Untersuchungen zur musiktheoretischen Fachterminologie

- Arbeiten zur Methodik und Vermittlung von Musiktheorie/Gehörbildung

- Erforschung der Beziehungen zwischen Theorie und Praxis (z. B. die Wechselbeziehung von Analyse und aufführungspraktischer Interpretation)

Anhand der aufgestellten Polarisierung in Kunst einerseits und Wissenschaft andererseits sollte jedoch nicht ein Rechts- oder Linksruck des Theoretikers in Richtung musikwissenschaftlicher Forschung gefordert werden; ebensowenig darf die Rolle des Theoretikers als Mittler zwischen beiden Polen vergessen werden. Vielmehr plädiere ich für eine durch Einführung des Promotionsrechts intensivierte musiktheoretische Forschung, die nicht als konkurrierender Gegensatz, sondern als komplementärer Ausgleich zu einer einseitig betriebenen praxisbezogenen `Pflichtfachkultur`verstanden werden will. Unter dieser Voraussetzung erscheinen die merkwürdigen Ängste und Vorurteile auf beiden 
Seiten überflüssig: etwa dem Theoretiker als Wissenschaftler mangelnde Praxisnähe (und manchmal auch mangelnde satztechnische oder analytische Kompetenz) vorzuwerfen oder umgekehrt den Künstler-Theoretiker mangelnder wissenschaftlicher Methodenkompetenz zu verdächtigen. Vergleicht man die deutsche mit der nordamerikanischen Theorielandschaft, kann man nur neidvoll auf deren institutionelle Verankerung und Ausstattung blicken: Musiktheorie ist als eigenständiges Fach mit selbstverständlichem Promotionsrecht anerkannt. Und erlebt man die Vorträge unserer angloamerikanischen Kollegen (wie man dazu auf dem diesjährigen Kongress der GMTH in München reichlich Gelegenheit hatte), so sind die Bedenken gegenüber praxisferner Theorieforschung absolut unberechtigt. Im Gegenteil gewinnt man schnell den Eindruck, als wären die promovierten Kollegen aus den USA weitaus praktischer und anschaulicher in ihren Vorträgen als so mancher Künstler-Theoretiker hierzulande. Bedenken gegenüber der Promotion in Musiktheorie sind daher völlig unberechtigt, ja sie erscheinen mir geradezu kontraproduktiv für unser Fach zu sein. Ich sehe in der durch Einführung des Promotionsrechts geförderten Aufarbeitung des noch weitgehend brachliegenden musiktheoretischen Forschungsgebietes (im Sinne einer die praxisbezogene Musiktheorie ergänzenden und befruchtenden, nicht sie ablösenden Betätigung) eine große, wenn nicht überlebenswichtige Zukunftschance für unser Fach.

\section{Alexander Rehding, Princeton}

Promotion in Musiktheorie? Eine ähnliche Diskussion kam hier neulich im kleinen Kreise zustande, da meine Universität keinen separaten Musiktheoriekurs anbietet (im Gegensatz, muß man hier einflechten, zur Konkurrenzuni, die zwei Staaten weiter nördlich liegt und mit $Y$ anfängt, die der amerikanischen Musiktheorie ihren Stempel aufgesetzt hat). Die mehrheitliche Meinung in unserem trauten Kreise war, daß das Modell, Musikgeschichte (musicology) nicht von Musiktheorie zu trennen, erfolgreicher sei als die strikte Teilung, die zu Spitzenzeiten der amerikanischen Musiktheorie in den 80er Jahren zu einer Art musikwissenschaftlicher Apartheid geführt hatte. Und obwohl sich seitdem viel an besagter Konkurrenzuni getan hat, hat sie es bis heute noch nicht geschafft, diesen Ruf ganz abzuschütteln. Es mag widersprüchlich erscheinen, daß ich einerseits den Dresdnern gratuliere und andererseits dem amerikanischen Musiktheoriebetrieb kritisch gegenüberstehe. Es ist dabei allerdings zu bedenken, daß sich Dresden am Anfang einer Bewegung befindet, die in Amerika seit fast einem halben Jahrhundert im Gange ist. Daß dabei sehr viele wichtige Arbeiten herausgekommen sind (die z. T. im deutschen Raum noch ihrer Entdeckung harren), läßt sich bei aller Kritik nicht bestreiten. Ohne den Alleingang der Musiktheorie, die Konzentration auf musiktheoretische Probleme in der Forschung, wäre dies sicherlich nicht möglich gewesen. Die Gefahr besteht nur eben darin, daß sich der musiktheoretische Diskurs verselbständigen könnte und sich - wie es in den 80er Jahren, in Anlehnung an den Wizard of Oz, angeblich hieß - als 'good witch der sbad witch Musikgeschichte gegenüberstellen könnte. Es fällt mir jedoch schwer, dies als reelle Gefahr im Rahmen des deutschen akademischen Betriebs zu sehen, da das intellektuelle Klima ein grundsätzlich anderes ist. Daher ist zu hoffen, daß 
das Dresdner Modell Schule macht und sich die Musiktheorie - als sequalı, aber nicht als ssame - etablieren kann. Weiterhin viel Erfolg!

\section{Gesine Schröder, Leipzig}

Schon vor 25 Jahren hat Dahlhaus in einer Glosse gewünscht, daß man »Musiktheorie beim Wort« nähme, als künstlerisch-wissenschaftliches Studium. Musiktheorie nun ein eigenes Promotionsfach neben Musikwissenschaft (und nicht nur ein Teilbereich): Vielleicht gelingt es der Musikwissenschaft so wie der Musiktheorie, die gegenseitigen Vorbehalte zu vergessen und Promotionen der anderen Fakultät anzuerkennen. Tonsatzlehrer müßten nicht mehr die Kolleginnen und Kollegen verdächtig finden, die Musikwissenschaft (zusätzlich zu Tonsatz oder Komposition) studiert haben, und die Musikwissenschaftler würden sich nicht scheuen, Erkenntnisse zu akzeptieren, die aus einem künstlerischen Umgang mit den Gegenständen erwuchsen.

\section{Thomas Seedorf, Freiburg}

Warum sollte man nicht im Fach Musiktheorie promovieren können? Bei dem Niveau, auf dem Musiktheorie mittlerweile betrieben wird, steht wohl außer Frage, daß Kriterien von Wissenschaftlichkeit, die für eine Promotion unabdingbar Voraussetzung sein müssen, gegeben sind. Wie sich Musiktheorie zur Musikwissenschaft verhält, wie beide Fächer miteinander kooperieren und koexistieren können, das ist sicherlich von Ort zu Ort unterschiedlich und immer sorgfältig zu erwägen. Ich fände es schade und überflüssig, wenn beide Disziplinen in Konkurrenz zueinander träten, schon deshalb, weil Prestigegerangel und Kämpfe um Finanzmittel in der Situation, in der sich das Land im Moment befindet, sicherlich niemandem nutzten. In diesem Sinne: Macht das Beste daraus!

\section{Oliver Schwab-Felisch, Berlin}

Dissertationen mit musiktheoretischem Thema werden künftig sowohl im Fach Musiktheorie als auch im Fach Musikwissenschaft geschrieben werden. Die Auswirkungen dieser Neuerung hängen nicht zuletzt von dem Verhältnis der beiden Fächer zueinander ab, einem Verhältnis, das durch die institutionelle und fachliche Konstitution des Faches Musiktheorie maßgeblich mitbestimmt wird.

Als eigener Studiengang wird Musiktheorie bis auf weiteres nicht an Universitäten, sondern nur an Musikhochschulen angeboten. Der wissenschaftliche und künstlerische Hintergrund des betreuenden Hochschullehrers und die jeweiligen Spezifika des Studienganges Musiktheorie werden die Physiognomie künftiger Dissertationen zweifelsohne mit beeinflussen. Entscheidender allerdings scheint mir, was Doktorand wie Betreuer als den disziplinären Rahmen ansehen, innerhalb dessen die Arbeit entsteht. Hier werden Gegenstände, Forschungsfragen und Diskussionszusammenhänge der Musikwissenschaft im selben Grad maßgeblich bleiben, wie es der Hochschul-Musiktheorie nicht gelingt, sich zumindest partiell als auch wissenschaftlich eigenständige Disziplin zu etablieren. 
Solange die Leitlinien musiktheoretischer Forschung von der universitären Musikwissenschaft gesetzt werden, beschränkt sich der Sinn des Promotionsrechtes im Fach Musiktheorie im wesentlichen darauf, die Hochschul-Musiktheorie am akademischen Diskurs zu beteiligen. So erstrebenswert und sinnvoll eine solche Teilhabe in verschiedener Hinsicht auch ist: Das Potential des Projekts Promotionsrecht verwirklicht sich erst, wenn drei weitere miteinander verschränkte Momente hinzutreten.

1. Musiktheorie versteht sich stärker als bisher als auch systematische Disziplin. Ohne die Formulierung, Diskussion und Weiterentwicklung aktueller Theorien und Methoden bleibt der Anspruch, den das Fach mit der Einführung des Promotionsrechts erhebt, in seinem Kern uneingelöst. Spezialisierung und Differenzierung, die Folgen aktueller Forschung, sollten als Preis der Professionalisierung und zugleich als Antrieb zu weiterer Theoriebildung verstanden werden.

2. Der musiktheoretische Diskurs wird wenigstens zum Teil in einem auch institutionell als musiktheoretisch definierten Rahmen geführt. Hierzu bedarf es eigener Organe, Strukturen und Medien. Auch nach Gründung der GMTH bleibt hier einiges zu tun.

3. Die Promotion im Fach Musiktheorie erlangt den Status einer gleichwertigen und aus fachlichen Gründen attraktiven Alternative zur Promotion im Fach Musikwissenschaft.

Die deutsche Situation unterscheidet sich von der amerikanischen grundsätzlich dadurch, daß der Lehrstuhl im Fach Musiktheorie auf absehbare Zeit eine Besonderheit der Musikhochschulen bleiben wird. Solange aber das Fach den Rang einer universitären Disziplin nicht erlangt hat, bleibt eine arbeitsteilige Ausdifferenzierung der Fächer Musikwissenschaft und Musiktheorie ebenso unwahrscheinlich wie eine Verlagerung bisheriger Schwerpunkte musikwissenschaftlicher Beschäftigung mit Musiktheorie in das Fach Musiktheorie. Keineswegs also droht eine Amerikanisierung des Fachs. Vielmehr werden Musikwissenschaft und Musiktheorie das Forschungsgebiet Musiktheorie gemeinsam und in Wechselwirkung miteinander weiterentwickeln. Produktiv wird diese besondere Konstellation aber erst, wenn die Hochschul-Musiktheorie den größtmöglichen Grad an Individuation anstrebt - eine Individuation, die durch eine kritische Auseinandersetzung mit dem Forschungszusammenhang, der die Eigenständigkeit des Fachs am weitesten vorangetrieben hat, dem angelsächsischen, nur befördert werden kann.

\section{Christian Thorau, Berlin}

Die Diskussion um die Einführung der Promotion im Fach Musiktheorie betrachte ich als große Chance. Bereits die Argumente, Entwürfe und Visionen, die bei der Auseinandersetzung um eine solche Einführung entstehen, können zum Katalysator für die Identität des Faches werden. Von den vielen bereits genannten Hinweisen halte ich drei für besonders bedenkenswert:

Dreieck der musikbezogenen Promotionsfächer: Die Promotion im Fach Musiktheorie ist als konsequente Weiterführung jener Entwicklung zu sehen, die sich in der Musik- 
pädagogik vollzog. Dort steht das Fach im Spannungsfeld zwischen Kunst und Pädagogik, und die Promotion bietet den Raum, die Phänomene und Probleme dieses Feldes wissenschaftlich zu reflektieren. Die Etablierung einer solchen Promotion geschah nicht umsonst dort, wo für Musik und für die Vermittlung von Musik ausgebildet wird: an den Musik- bzw. Kunsthochschulen. Mit dem Dr. phil. im Fachgebiet Musiktheorie verhält es sich parallel: Dort stehen die Phänomene und Probleme im Spannungsfeld zwischen Kunst und Wissenschaft, und die Promotion reflektiert dieses Feld mit wissenschaftlichen Mitteln. Und das geschieht am besten dort, wo dieses Spannungsfeld am stärksten und vielseitigsten ist. Hier könnte die Profilbildung des Faches einsetzen: Promotionen in Musiktheorie sind solche, die sich in besonderem Maße mit dem Spannungsfeld zwischen ästhetischer Praxis (Komponieren, Aufführen und Hören) und ihrer Theoriebildung auseinandersetzen. Tatsächlich lassen historische und systematische Musikwissenschaft hier vieles unberührt.

Keine neue »Apartheid«: Die amerikanische Warnung vor einer Wiederholung der (Fach)Geschichte ist ernstzunehmen. Den Umweg über die Abspaltung des Faches mit allen Nebenwirkungen der Isolation und Gettoisierung sollte man sich sparen, zumal während der gegenwärtigen Umstrukturierung des Hochschulsystems. Dafür ist die Musiktheorie auch schon viel zu integriert und wissenschaftsfähig. Die Einführung der Promotion in Musiktheorie darf den Dialog mit Musikwissenschaft und Musikpädagogik nicht abbrechen lassen, sondern muß ihn im Gegenteil aufnehmen und intensivieren. Ein gegen den Widerstand dieser Fächer ins Leben gerufenes Promotionsrecht ist retrospektiv und nicht viel wert.

Nicht formal denken, sondern inhaltlich: Nicht der Dr. phil. in Musiktheorie ist entscheidend, sondern, ob von musiktheoretisch ausgerichteten Dissertationen inhaltiche und methodische Impulse ausgehen. Diese sehe ich weniger in der Spezialisierung als in der Öffnung des Faches. Wer sich fragt, in welchem Verhältnis die musikalischen Gestaltungsformen, die man unterrichtet, zu jenen Ästhetiken stehen, die die Wahrnehmung außerhalb des geschützten Raumes einer Hochschule prägen, hat mehr mit den Fragen der Musikpädagogen gemeinsam als mit denen vieler Musikwissenschaftler. Und wer einmal mit dem Dozenten für visuelle Grundlehre am (benachbarten) Fachbereich Design kooperiert hat, merkt, wie spielend Musiktheorie als ästhetisches Grundlagenfach die Kunstgrenzen überwindet. Solche interästhetischen Verbindungen lassen sich mit wissenschaftlichen Mitteln reflektieren, und es spricht vieles dafür, daß das Haus der Musikwissenschaft dafür nicht groß bzw. nicht offen genug ist. 